\title{
Correction to: Percutaneous retrieval of migrated Viabahn stent from a segmental pulmonary artery
}

\author{
R. C. Zvavanjanja
}

\section{Correction to: CVIR Endovasc \\ https://doi.org/10.1186/s42155-018-0007-3}

In the published article (Zvavanjanja 2018) the statement under the subheading 'Consent for publication' is incorrect.

"Retrospective anonymized and no patient photos therefore no institutional requirement."

should read:

"Informed consent for publication of this case report and its accompanying images has been obtained from the patient"

Published online: 06 January 2020

\section{Reference}

Zvavanjanja RC (2018) Percutaneous retrieval of migrated Viabahn stent from a

segmental pulmonary artery. CVIR Endovasc 1:1 https://doi.org/10.1186/

s42155-018-0039-8. https://doi.org/10.1186/s42155-018-0007-3

\footnotetext{
Correspondence: Rodrick.c.zvavanjanja@uth.tmc.edu

The original article can be found online at https://doi.org/10.1186/s42155018-0007-3

Department of Diagnostic and Interventional Imaging, University of Texas

Health Science Center at Houston, McGovern Medical School, 6431 Fannin

St, MSB 2.132, Houston, TX 77030, USA
} 